\title{
AN ENHANCED WIRELESS NETWORK FOR LOW INCOME USERS
}

\author{
Edim, Azom Emmanuel \\ Department of Computer Science, University of Calabar \\ Calabar, Nigeria \\ edimemma@gmail.com \\ Ogban, Felix Ukpai \\ Department of Computer Science, University of Calabar \\ Calabar, Nigeria \\ fogban@gmail.com \\ Ofem Ajah Ofem \\ Department of Computer Science, University of Calabar \\ Calabar, Nigeria \\ Ofem_ajah@gmail.com \\ Okoro, Osahon \\ Department of Computer Science, University of Calabar \\ Calabar, Nigeria \\ osahonokoro@yahoo.com
}

\begin{abstract}
Information and communication technology present huge potentials for socio-economic development in under-served communities. Wireless network offers great opportunity for the provision of affordable Internet services and bridge the digital gap among low income earners. In this study, a wireless network that is scalable and easily manageable was designed and implemented to cover five rural communities. We have been monitoring and maintaining the network for more than three years. Over time signal attenuation has been experienced as a result of environmental changes such as trees creating obstacles. A mesh network at the access layer was deployed to enhance throughput and signal propagation. Data was collected and analyzed to assess network and end users performance. The results shows that the transmit/receive $(\mathrm{Tx} / \mathrm{Rx})$ rate of $300 \mathrm{Mbps} / 300 \mathrm{Mbps}$ was observed and a throughput loss of $0.5 \mathrm{Mbps}$ on the network. The point-to-multipoint access layer design increased the throughput, enhance signal coverage and quality of service.
\end{abstract}

Keywords: Wireless network; Point-to-multipoint; Internet service.

\section{Introduction}

The Internet has remained popular and has gained tremendous reputation due to the huge wealth of knowledge or information it has provided to its users across the world. The Internet has remained influential in every sector of our socio-economic life. In most developing countries like Nigeria, access to Internet services has continued to develop from one form of user access method to the other. These include the cyber café operators who acquired bandwidth via very small aperture terminals (VSAT). The bandwidth was costly and as such relatively low. Users visit the cyber café and were billed by a time-based billing system that enables the operators to get their return on investment. This meant that users will have to spend a lot of time surfing the web and Internet services in the cyber café to make meaningful research or Internet usage. Internet connectivity became more robust and popular with the introduction of the fixed wireless broadband service since 2004 till date. This enabled users with wireless equipment send wireless Internet connection to their homes or business locations through wireless Internet service providers (WISP) who were found almost all in the major cities in Nigeria.

The establishment of the mobile telecommunication network system enabled the use of mobile devices that gradually provided easy-access to Internet services in the urban areas. Modems were also introduced by the GSM companies and this provided little or limited bandwidth to the users in the urban centres for Internet connectivity with laptop computers. Subsequent upgrade of GSM networks from $2 \mathrm{G}$ to $3 \mathrm{G}$ and to $4 \mathrm{G}$ was performed as a result of the increasing demand for bandwidth and Internet connectivity. This increase in demand has also prompted the mobile network operators to lay fiber cables to their base-stations. The increase in the number of smart phone users in urban communities means that more users are now having mobile Internet access for educational and socio-economic development. 
The rural communities in Nigeria have a good number of mobile subscribers, but Internet access remains a problem due to many factors such as low literacy, poverty, high cost and poor infrastructures. Internet providers consider these communities as none viable because of the fear of not being able to get the required return on investment. And so these communities remain disconnected from the rest of the world and lack Internet service for socio-economic development. The traditional wire-line connection solutions as broadband, fiber, and dial-up are not economically suitable for deployment in rural communities as a result of high cost to build and low user densities (Brewer et al., 2006; Du et al.. 2006; Mishra et al.. 2005). Due to the terrain in the rural communities, Satellite networks can be used to achieve high coverage. This option is effective but too costly to implement considering the population, high cost of deployment and expected return on investment. An ISP rate of $1 \mathrm{Mb} / \mathrm{s}$ of satellite connectivity from satellite network can be very costly and for the mobile telecommunication network providers this high speed Internet service can only be suitable for urban users who can afford it.

In recent years, the cellular revolution has brought significant penetration of cellular networks in rural communities. The cellular networks have deployed broadband network technologies such as GPRS, WiMax, and CDMA (Nedevschi. et al., 2007). Mobile phone users in rural communities use their devices mostly for telephony services and very few use their devices for limited Internet services due to high cost of service which are unaffordable (Yunus. 1999). This means that Internet services for socio-economic development will continue to elude the rural dwellers. Services such as education, e-health, e-banking, e-commerce, e-government and agricultural information through the Internet remain unaffordable and not accessible. Due to low user density in rural areas an economically viable Internet connection solution will require small per-user setup cost and low recurring cost (Subramanian et al.. 2006: Mishra et. al.. 2005). It has been discovered that in rural areas, high network operation cost are incurred from power consumption due to large coverage area, use of backup power system, and high cost of securing the network infrastructure (Mishra et. al., 2005).

Information and Communication Technology (ICT) provides a platform that can be used to create economic activities that can improve the wellbeing people living in rural communities (Edim and Muyingi, 2013). ICT can boost agriculture, education, health care, socio-cultural development and alleviate poverty. The Internet provides access to information on agricultural practices through agriculture extension service, a platform for sale of farm produce, craft works (Edim et al., 2013) and as well as other economic ventures that are common with rural people.

The Institute of Electrical and Electronics Engineers (IEEE) has provided a number of technologies as standards for ICT hardware deployments for Internet services. The IEEE "802" standard has high capacity for different network connectivity providing affordable variable-size network packets and services. The IEEE 802.11 standard supports quality of service at the MAC level in Wireless Local Area Network (WLAN). The IEEE 802.11 WLAN compliant operate in the $2.4 \mathrm{GHz}$ frequencies and provides a high transmission rate up to 54 Mbps. The 802.11 standard had been used to configure Wireless Ad hoc network and infrastructure providing reliable and enhanced network connection (Netgear, 2005). This can be extended in the rural communities to provide network and affordable Internet services.

In rural communities wired-line network are not viable because of the terrain. Wireless Local Area Networks (WLAN) for communication and Internet services can be deployed with a set of Media Access Control (MAC) and physical layer (PHY) specifications with frequency bands of 2.4. 3.6. 5 and 60GHz. The 802.11 standard provides wireless link and data encoding with the direct-sequence spread-spectrum (DSSS) technology. The DSSS enables transmission rate up to $11 \mathrm{Mbps}$ and takes a signal and spread it over a wide frequency band for transmission (Gast, 2005). This is transmitted using the unlicensed radio spectrum at 2.4GHz. While the IEEE $802.11 \mathrm{i}$ standard enhances wireless encryption and security in a WLAN. In the rural area, any successful network deployment will depend on security, scalability, sustainability and cost effective infrastructures (Disraeli, 2001). We have deployed a Wireless network infrastructure for more than three years now and have continually been monitoring, maintaining and improving on it to make it sustainable. We have improved on the connectivity between access point and user end devices by introducing a mesh network on the access layer of the network using the ubiquity hardware. The network infrastructure was developed using technologies that are cost effective, scalable and secured. It has been providing affordable Internet access with high quality of service to users in rural communities. One major challenge in the communities is lack of adequate power supply from the national grid. But the installation of backup power with solar cells and batteries we have ensured that the network remain operational. This paper presents the implementation of the wireless network and the services provided to the host communities.

\section{Literature Review}

The rural communities also need affordable Information and Communication Technologies for socioeconomic development. They need to also communicate and reach out to people living outside their communities. Mobile telecommunication operators have taken mobile telephony to the rural areas for voice communication, but they remain reluctant to increase their investment in deploying network infrastructure in 
many remote areas (Dymond and Oestmann, 2003). Most rural areas in Africa have limited or no connectivity of cellular network. In these areas, installation of cellular infrastructure is very complex and requires the erection of very tall Base Stations (BTS) and other necessary infrastructures. The cost of installing and maintaining these infrastructure is very high and makes it difficult for the operators to establish a network that will yield return on investment in rural communities with low income and low population densities. With a population that is characterize with seasonal revenue from subsistence farming, rural users often use prepaid tariff unevenly throughout the year due to unsteady source of income (Flickenger et al 2006: Horst and Miller, 2006). This makes the use of cellphone very expensive to rural users and even more if they are to apply their devices to purchase data for Internet access.

Technological advancements in Wireless Local Area Network (WLAN) infrastructures has provided opportunities for sustainable Internet connections in rural areas (Galperin, 2004). This has boosted the installation of low cost wireless devices in low density and low income communities. With this development, Internet services can be made available to remote communities with small population and with low income. Asynchronous wireless systems can be used to offer broadband connections, local data caching for long-distance backhaul, while also maintaining local infrastructure in the communities (Pentland, Fletcher \& Hasson, 2004). The wireless low-cost systems sustainable, easy to maintain and are scalable and sustainable. These are good options for meeting ICT demands in rural communities.

Wireless technologies operate on different economy of scale as compared to wired technologies. The new WLAN technologies have created room for bottom up infrastructure development by different actors such as different agencies and user cooperatives. Sustainability is possible with small scale deployment and local actors can actively be involved during development and use of ICT services. This enable the network to be more closely aligned to the local context and community needs and can easily be expanded.

Large ICT deployments are expensive and require huge capital from government agencies and big telecommunication operators (Galperin, 2005). Only these entities had the capital to achieve the required economy of scale involved in laying down the required coper wires and other infrastructures, as well as the high capacity switching equipment for the network (Noll, 2000). However, advances in wireless technology has brought cost reduction and enabled the setting up of wireless solutions even where the terrain is bad and no legacy wired network is found, especially in rural communities.

Developments in WLAN has created opportunity for providing Internet services in developing countries which could surpass what the cellular network for basic telephone services has done. WLAN technologies such as the IEEE 802.xx standards are non-cellular wireless infrastructures for communications. They consists of a broad spectrum of equipment with variety of attributes. The family of IEEE 802.11 standards known as Wi-Fi was selected for implementation in this study. These standards are widely accepted and have been applied in the deployment of WLAN infrastructure in different regions. There are a lot of challenges that comes with the deployment of network infrastructure in remote communities such as security of services, network maintenance, weather, RF pollution and regulations that may change from time to time. For the internal security of network services, the IEEE 802.11i standard provides protocols to regulate and enhance wireless encryption and provides enhance security for the network. This facility has been deployed recently as part of the network scalability and maintenance plan to enhance the security of user data and services.

\subsection{Internet Landscape in Rural Communities}

Despite the wide spread of Telecommunication infrastructures in developing countries, Internet services in rural communities remain poor and costly. Internet connectivity in developing countries remains at a very low percentage, and even at that, this percentage is mostly found within the urban communities (World Bank, 2004). Wide spread poverty in rural communities limit the deployment of telecommunication infrastructure and Internet services. Rural communities with mobile telecommunication presence often times experience poor quality of service for voice calls. Internet services are even more difficult to access. This is as a result of the cost associated with the implementation of telecommunication infrastructure with good quality of service. On the other hand, cost of leased-lines, international connectivity and government regulations have contributed to the deployment of network infrastructure that provide limited data capacity, high prices and low service demand (Wallsten, 2003). There is therefore a need to review the infrastructure landscape in order to implement technologies that will play down on the low population densities of rural communities, reduce the cost of implementation and provide quality services to users in rural communities. WLAN technologies have improved tremendously to provide sustainable wireless solutions, with relatively cost-effective network deployment in rural communities. Obviously, this requires the involvement of industry technical players such as computer programmers, engineers, network developers, and energy engineers for the implementation of a network that is sustainable and that is tailored to the local context and meets the needs of the communities. 


\subsection{Network Infrastructures}

In remote communities, the mobile communication (GSM) networks provides voice communication as well as content and information to the inhabitants (Kumar et al., 2007; Patel et al.. 2010). The Global System for Mobile communication (GSM) network is the common technology for providing Internet services to the people. The GSM technology incorporated other types of technologies for it to perform properly. These technologies include the High-Speed Circuit-Switched Data (HSCSD), Enhanced Data for GSM Environment (EDGE), Universal Mobile Telecommunication System (UMTS), and General Packet Radio System (GPRS) (Nikos et al., 2010). Although the manner in which these technologies are applied depends on the customs and norms of the people (Horst and Miller. 2006). Apart from this, these technologies may provide low quality of service. Also, third-party remote server required to drive voice communication with a single gateway network on the Internet can also create bottleneck when multiple request are made on the network server. Wireless Fidelity (Wi-Fi) was conceived as a technology to connect computers within limited distance. The technology has evolved over the years to also connect devices in public places and has further been upgraded to connect long distance devices with point-to-point communication.

\subsection{Wireless LAN Infrastructures for Rural Communities}

Technological improvements in the 802.11n standards has enhance the throughput of wireless network connectivity to nearly $200 \mathrm{Mbps}$. This speed of transmission is twice as fast as the fast Ethernet of $100 \mathrm{Mbps}$. If deployed in rural communities, it has the capacity to provide fast speed Internet connection to rural communities. Wireless LAN have been used to provide network services in different places, but mostly in urban centres. Long et al. (2012) describes a Wireless LAN videophone to improve the safety of video communication for network cameras, residential surveillance and traffic monitoring. This system used sophisticated and costly network infrastructures for its implementation. Imran and liaquat (2007) implemented a wireless network in the computer department to complement the existing local area network. The system enhanced access sharing and exchange of information among the users. The authors upgraded from the standard IEEE $802.11 \mathrm{~b}$ to IEEE $802.11 \mathrm{~g}$ in order to enhance the security and information access. Toni and Wendy (2007) describes the design and implementation of a wireless LAN in a University campus. The network provided all users with mobility, meaning that they could access the network everywhere on the campus. They used a mathematical model to predict the access point coverage. The network was useful for improving the quality of education and administrative work flow in the school. The above studies were successful and concentrated in places that did not have so much environmental challenges that hinder signal propagation between access points and user devices.

Technologies such as the MIMO (Multiple input, Multiple output) are standard equipment that send multiple signals carrying different data sets through different paths thereby reaching out to users spread across communities. Enhancements in Wireless access points has simplified connections with user devices containing wireless cards that are $802.11 \mathrm{n}$ compliant. WLAN computer communications within the frequency band of 2.4, 6.5 and $60 \mathrm{GHz}$ uses the IEEE 802.11 protocol stack. The Media Access Control (MAC) and the Physical Layer (PHY) specification are within the IEEE standard protocol (IEEE, 1999). In long distance signal transmission networks covering large expands of space such as in rural areas, the 802.11 is used to manage collisions in a broadcast multi-access. In such networks long propagation delays are common (Sonesh et al. 2008).

The rural vision center network is an implementation of a WiFi-based Long Distance network (WiLDNet) (Patra et al., 2007) as a means to overcome the drawbacks in the 802.11 MAC protocol. With TDMA-based MAC protocol, WiLDNet was able to recover from data loss and met the requirements set up for rural users. Other rural networks based on the WiLDNet include the Airjaldi network, and the Aravind telemedicine project (Sonesh et al., 2008). These networks had good throughput in their performance, but were limited in the distance of communication. Also attenuations were common as a result of interference from obstacles within the environment. The WiLD MAC became an enhancement of the WiLDNet and was developed to overcome high packet collision in long distance transmission links associated with the 802.11 standard protocol. With fixed time slot method between end-points adopted by WiLD MAC it was able to overcome the stop-and-wait rule that was prevalent in the 802.11 protocol. The drawbacks of WiLD MAC include low throughput transmission. Further modification of the WiLD MAC led to the introduction of adaptive time-slots to replace the fixed time slots. This enhanced version was initially called "JazzMAC" and later known as the WiRE (Divya, 2011). WiRE adopted the adaptive time slot in the TDMA-based protocol which had capability to adapt to the different traffic demand. WiRE implementation had problems with environmental obstacles that affected connectivity between access points and user end devices. Long distances between access points and user devices led to high signal lost. The Wifi-based wireless network that was implemented considered environmental obstacles, used a point-to-point/multipoint (P2PMP) network structure as an enhancement on the WiRE (Divya, 2011). In this our implementation, access points were located at points 
that were closer to the users and their end devices in order to increase the signals between access points and the user devices or the network throughput. This has further been enhance by implementing a mesh network within the access layer to mitigate the effect of the changes in the environment that had increased interference on signals and reduced the signal strength getting to the end users. The mesh network has enhanced the signal and the network for better throughput.

\section{Research Method}

\subsection{Data Collection}

The network covers five locations/villages and has been on operation for more than five years. During this period, we have been monitoring, maintaining and gathering data on network performance for evaluation. The processes include regular on-site visits, interactions with technicians and end users for data collection and evaluation to determine network performance. The redesign process involved environmental survey to identify new obstacles like trees, buildings and mountains along the transmission path. The next phase was to redesign the network by implementing the mesh network at the access layer. This addition is a proof of network scalability. The onsite visitations and survey of the environment was carried out in five different communities which include Ezzamgbo, Azuiyi Udene, Ntezi Abba, Ezza, and Ishieke. The wireless mesh structured network was designed to cover these five locations. In the network, ten paths for wireless links were identified. The distances between the sites, line of sight for each link and the geo-coordinates of all the sites were determined using the magnetic compass. The values were inputted into the google earth software "Ruler tool". Other tools include binoculars, 14 pixels sony digital camera and writing materials. Interviews were also conducted with the potential users of the network. The $802.11 \mathrm{n}$ chipsets and devices were chosen for the design because we wanted the network to deliver high throughput, availability and data security (LitePoint, 2012). A template was created containing criteria for network validation based on the requirement specification. These criteria include Availability check, Security check Scalability check, manageability check, Availability check, ambiguity checks error and inconsistency checks. The Microsoft visio design tool was used for network design. In the design, the network was segmented into three layers: the core, distribution, and access layers. We adopted the Cisco hierarchical network model to structure the design.

\subsection{Network Design and Implementation}

The Cisco network design model was applied with adjustments to realize the system design. We considered the five locations that are geographically dispersed to implement a multiple network design. The design consists of a hierarchy with the core layer at the top, the distribution layer at the middle and the access layer at the bottom.

The core layer formed the core of the network. It is responsible for transporting large amount of traffic reliably and quickly. It is majorly responsible for switching traffic as fast as possible. At the core layer a 2.6 diameter dish VSAT (very small aperture terminal) (outdoor/indoor) antenna was installed for Internet connection. A bandwidth of $26 \mathrm{mbps}$ download and $4 \mathrm{mbps}$ upload was the specification for wireless distribution. Bandwidth shaping and authentication setup is done by the MikrotikRouterBoard 2011L router that was connected to the Vsat modem. The radius server and user database (MySQL, MyPhPAdmin, Dalaradius) were configured such that the router could monitor and control the user access. This layer is responsible for reliably transmitting large volume of data at high speed.

The distribution layer consists of the hardware and software parts and it links the core layer to the access layer. The hardware include the ubiquity NanoBeam M5 MiMO radios used as a point-to-point wireless connection of all the locations/communities. The network remote broadcast are bounded together using the Mikrotik 750GL. The 750GL routers contain the open shortest path first algorithm (OSPF)/software that is used to find the best path for trafficking packets at any given time. We configured virtual tunnels to enable packets move from each location to the core router. The distribution layer serves as the work group layer handling packets routing, filtering and WAN packets access within the core network (Todd, 2007).

The access layer is a Point-to-multipoint uplink structure with the ubiquiti NanoStation M5 (Access point) located at the base station. The ubiquiti NanoStation M5 Loco (Atel Electronics, 2015) with a Unifi UAPPRO 2.4Ghz were used at all the access points covering the network radius. All the locations serves as hotspots. The Ubiquity M5 Loco is used as Uplink to connect each hotspot. The Ubiquity UniFi was used to setup the access point at each hotspot. The configuration of the Ubiquity UniFi, device controller software created a wireless distribution system that allows all the hotspots to have a unique and identical service set identity (SSID). The OSPF cloud bridges the entire core routers into a virtual switch. Each access layer router is connected to a virtual switch with a configured control access point manager which provides the virtual service set identifier (SSID) used on the entire network. Every user end device is connected to the SSID. 
The Core layer is configured to stand as the Internet backbone providing the needed security in terms of the setup accounting, user authentication and the required policies to control all access to the network. The Distribution layer provides reliable and fast speed Internet services in each of the five locations. The distribution of the Internet services to the users is handled by the access layer. The overall architecture of the wireless network with all the three layers put together as a single network structure is presented in fig. 1 .

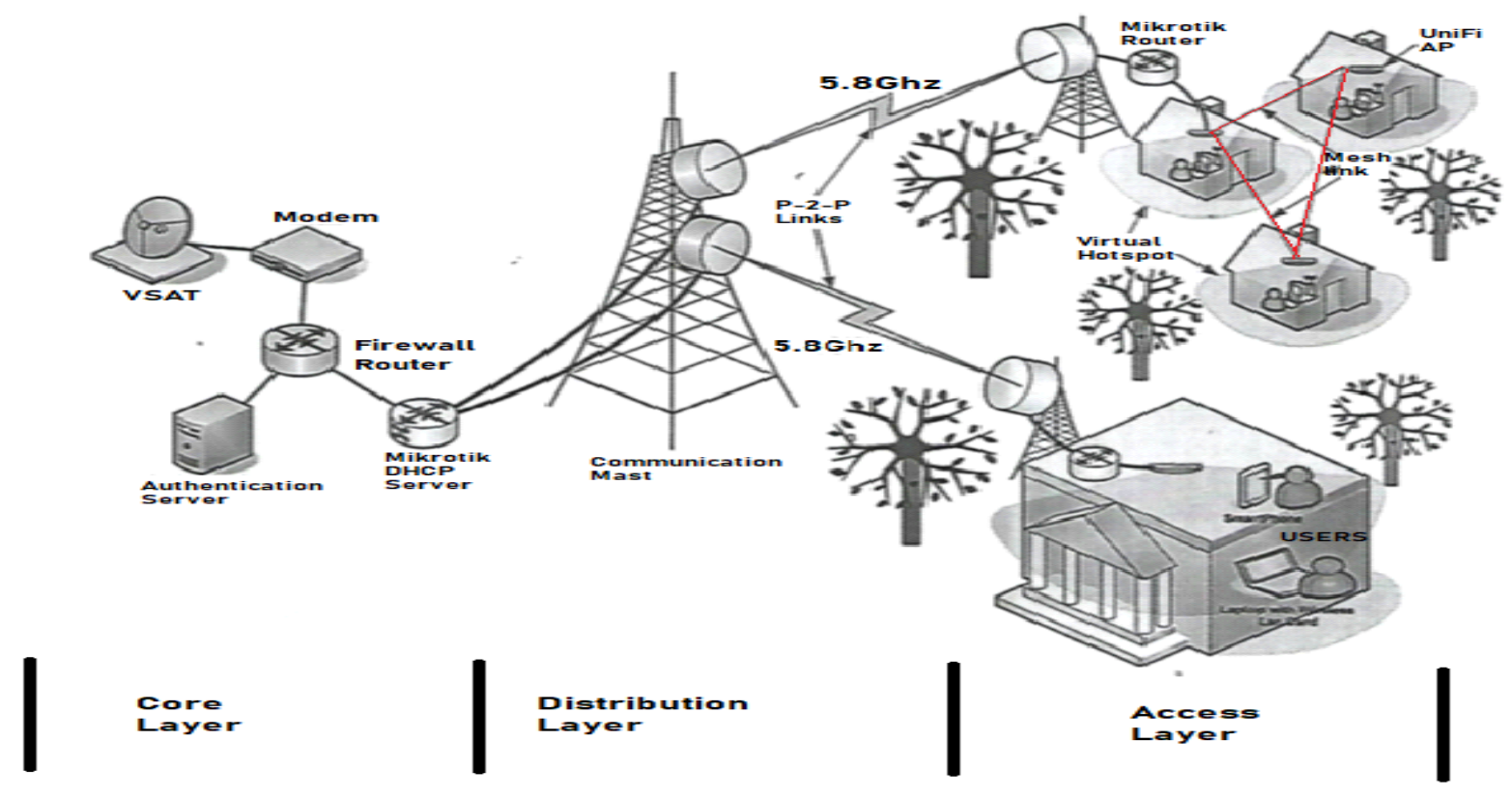

Fig. 1. The architecture of the wireless network.

Every conventional wireless network design is based on the hub-and-spoke structure whereby a router/L3 device sits at the top (core) and a switch network sit at the middle, leaving the access point to be located at the access layer. New challenges have emerged within the environment such as natural and man-made obstacles causing increase in blind spot within the network. Blind spots are areas where signals are very low for any form of connection with user end device to be meaningful. One way to address this challenge was to scale up the network by deploying more access points to give a complete coverage, but this option was more costly to realize. So we utilized a better option by using the controlled access point system manager (CAPSMAN) to develop a mesh network in the access layer using the ubiquity hardware. With this in place, if there is any obstacle between the access point and the user end device, the access point automatically calculates and finds the next best route to reach the user end device with strong signals. The security of the network has been upgraded using the IEEE 802.11i standard which enable data communication between the user end devices and access points in encrypted format. The IEEE 802.11i standard is a security enhancement protocol for wireless equipment privacy (WEP)-based security.

\subsection{Network Testing and Evaluation}

The entire network devices were tested in each location for signals and connectivity. User devices which include smart phones, tablet and laptop computers were also used to test the network. We had positive results during the testing. The network activities are monitored and tracked using the radius server installed with filters. Users have been using the network for various Internet services. We have been collecting data from the network as well as the users for more than five years now while monitoring and tracking activities in the network. The data analysis is carried out and results studied to determine network performance especially with the addition of the mesh network on the access layer.

\section{Data Analysis and Result}

We collected both quantitative and qualitative data during the processes that led to network design, deployment and monitoring. The data analysis and results are presented next.

\subsection{Implementation Site Data Analysis}

This study applied the magnetic compass to get the exact geo-coordinates of the selected network sites in the five locations. The geo-coordinates of Azuiyi Udene were $6^{0} 19^{\prime} 25.11^{\prime}{ }^{\prime} \mathrm{N}, 8^{0} 07^{\prime} 39.92$ ' 'E, that of Ezza 
were $6^{0} 19^{\prime} 15.31^{\prime} \mathrm{N}, 8^{0} 05^{\prime} 05.10^{\prime}$ 'E. The other locations include Ntezi Abba with geo-coordinates $6^{0} 19^{\prime}$ $16.57^{\prime}$ 'N , $8^{0} 05^{\prime} 31.62$ ' 'E; Ezzamgbo: $6^{0} 23^{\prime} 57.28^{\prime}{ }^{\prime} \mathrm{N}, 8^{0} 00^{\prime} 43.79^{\prime}$ 'E, and Ishieke: $6^{0} 22^{\prime} 38.21^{\prime}{ }^{\prime} \mathrm{N}, 8^{0} 02^{\prime}$ $34.28^{\prime}$ ' $\mathrm{E}$. The coordinates were inputted into the google earth software and the paths for the wireless signals were mapped out. The different routes and relative distances between the various points of interest or sites were also determined using the google earth software. The cluster of settlements, vegetation and land marks such as mountains were also mapped out. This information was very useful as it assisted in determining appropriate positioning of the access points. There have been changes in the environment which has further necessitation changes in the network structure with the addition of a mesh network at the access layer of the network.

\subsection{Wireless Network Performance Data and Analysis}

The wireless network is a point-to-multipoint (PtMP) connection at the access layer. The basic setting of the Nanostation M5 equipment as depicted in Fig. 2 indicates that it is set to "Access point" and the network mode configured as a "bridge". This is why the network traffic from the wired LAN interface is connected directly to the wireless LAN interface. The output in fig. 2 also shows that a channel capacity of $40 \mathrm{Mhz}$ handles interference on transmitted signal using a dynamic frequency selection (DFS) of $5560 \mathrm{Mhz}$. There was a reduction on the transmit power and a boost on the signal received by the antenna as a result of the configuration of the $2 \times 2$ special diversity chain. The result (fig.2) shows a reduced noise floor of $-92 \%$ which enhanced the quality and capacity of the AirMax. The AirMax is a proprietary protocol, an ubiquity Time Division Multiple access (TDMA) that allows each client to send/receive data using pre-assigned time slots organized by an intelligent AP controller. The throughput graph in figure 2 indicates the effect of the bridge between the wired LAN and wireless LAN interfaces. The graph shows that the signal transmit (Tx) in Wireless LAN interface correspond to the signal receive $(\mathrm{Rx})$ in the LAN interface. The graph is with a scale 0 to $3.5 \mathrm{Mbps}$, and a throughput of $1.5 \mathrm{Mbps}$ on the average was chosen as the required throughput at the end users devices which is indicated by the arrow on the graph. The result shows improvement on the quality of the signal send and received by end users irrespective of their location or environment characterized by different obstacles. The additional mesh network on the access layer ensures that the end user location or position does not affect the quality of the network connection. With this, the speed of data transmission remains high and with good quality of service.

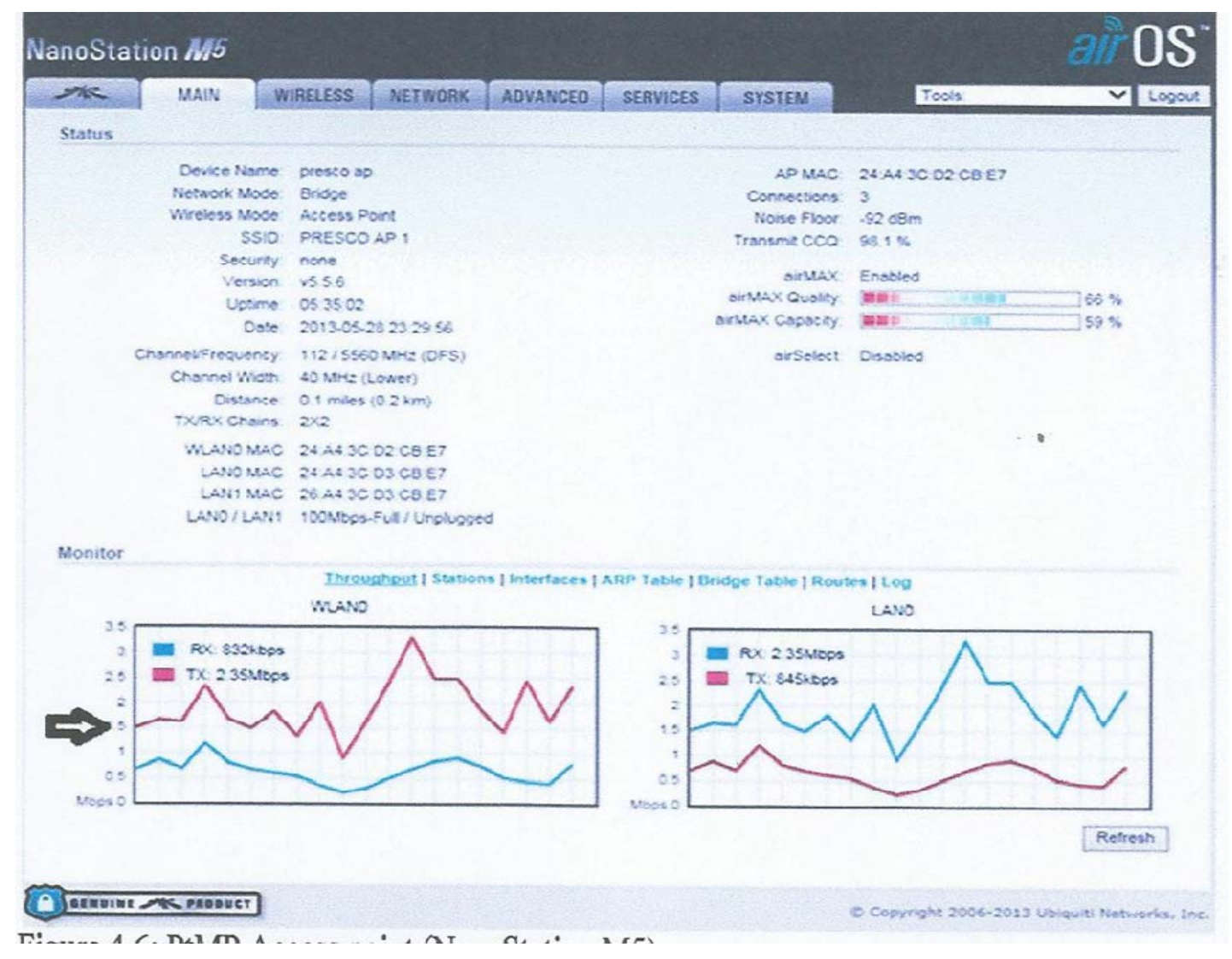

Fig. 2. PtMP Access Point (NanoStation M5) Output 
Table I presents the results from the network the main status of the user and network station devices. The result shows that the network achieved a signal strength of $-60 \mathrm{dbm}$ with a noise floor of $-90 \mathrm{dbm}$. The transmit/receive $(\mathrm{Tx} / \mathrm{Rx})$ rate of $300 \mathrm{Mbps} / 300 \mathrm{Mbps}$ was recorded which is thrice as fast as Ethernet (100Mbps). The monitor section on figure 2 shows an average throughput of $1 \mathrm{Mbps}$ which indicates a throughput loss of $0.5 \mathrm{Mbps}$ on the network. Each base station was able to transmit $100 \mathrm{Mbps}$ Ethernet speed into the $300 \mathrm{Mbps}$ wireless network effectively and this in turn was also transmitted to the user end devices through the $2.4 \mathrm{Ghz}$ wireless access network which used the Ubiquity $2.4 \mathrm{Ghz}$ UniFi access point. Each based station (BTs) was able to transmit these amount of data and speed (table 1). The results indicate a high quality and speed of signal transmission including the signals received by the end user devices. The system recorded $3 \mathrm{~GB}$ data per user per month which reflects the quality of service from radios protocol and quality of AirMax. With a performance level above 50\% the wireless network has demonstrated high performance and quality of service.

\begin{tabular}{|l|l|l|l|}
\hline \multicolumn{4}{|l|}{ PtMP NanoStation Loco M5 - Main Status /Output } \\
\hline Device Name & Presco Access Point 1 & AP MAC & 24:A4:3G:D2:C0:E7 \\
Network Mode & Bridge & Signal Strength & $-60 \mathrm{dBm}$ \\
Wireless Mode & Station & Vertical/Horizontal & $-63 /-63 \mathrm{dBm}$ \\
SSID & PRESCO-AP1 & Noise Floor & $-50 \mathrm{dBm}$ \\
Security & None & Transmit CCQ & $99 \%$ \\
Version & V5.5.6 & Tx/Rx Rate & $\mathbf{3 0 0 M b p s / 3 0 0 M b p s}$ \\
Uptime & $05: 30: 26$ & AirMax & Enabled \\
Date & $2021-01-20$ 20:25:20 & AirMax Priority & None \\
Channel/Frequency & $112 / 5580$ MHz (DFS) & airMax Quality & $67 \%$ \\
Channel Width & $40 \mathrm{MHz}$ (lower) & airMax Capacity & $66 \%$ \\
Distance & 0.1 miles (0.2km) & \\
TX/RX Chains & $2 \times 2$ & \\
WLAN 0 MAC & $24: A 4: 3 \mathrm{G}: 36: E 0: 4 C$ & \\
LAN 0 MAC & $24: A 4: 3 \mathrm{G}: 37: E 0: 4 C$ & \\
LAN 0 Bandwidth & $100 \mathrm{Mbps-Full}$ & \\
& \multicolumn{3}{|l}{} \\
\hline
\end{tabular}

All the access points were concurrently configured using the UniFi AP which is able to provide information on the number of end users that are connected to any given access point per time and the bandwidth (download/upload) being used on each access point including the $2.4 \mathrm{Ghz}$ channel frequency. The wireless network implemented in this study has been providing Internet connection with expected quality of service to the end users in the five locations. It has impacted positively on the communities by taking the users to the outside world, with new kinds of interactions and access to information.

We have been monitoring and collecting data from internal recordings of the network terminals since the deployment of the network. The lowest number of users (30 clients) was recorded in the month of July 2014. This was at the early deployment stage. After this time we have recorded increments both on the number of users and the data consumption rate. On one access point 165 users were observed using a bandwidth of 60.2 download and $4.0 \mathrm{G}$ upload. This observation demonstrate the capacity of the network to accommodate large number of users with high data transmission rate, at low cost compared to the normal GSM network (Nikos and Kyriako, 2010). The number of users has increased tremendously to an average of 250 per day in 2019 and 300 per day in January 2021. This was due to the expansion of the access point. The amount of data consumed per month has also increased. At the initial stage, in the month of July, 2014 a total of $3900 \mathrm{Mb}$ of data was consumed by the users.

This implies that for a single user, the average per month was approximately 3GB. This is a window for successful running of application that can add value to the community. An e-learning platform can successfully run with a bandwidth of $100 \mathrm{Mb}$ to benefit the students and pupils in the community on daily basis. With data access rate of $3 \mathrm{~GB}$ per user at very low cost, the Internet connection will be affordable to users in rural communities. The data rate has increased in 2019 to $750,300 \mathrm{Mb}$ and $920600 \mathrm{Mb}$ in 2021 . The Internet connection has been consistent hence the continuous increments in the data consumption rate. The increment in the number of end users means that the users consider the wireless network affordable and reliable. The high 
data rate experienced in the network is as a result of the mesh network of access points in the access layer placed closer to the end users and their devices, thereby reducing signal attenuation and lost due to interference from the environment. It will continue to add the value to their educational, social and economic life.

\subsection{Measures for Network Sustainability}

In designing the wireless network measures to enhance the sustainability of the network were incorporated into the design and were actualized at the implementation of the network. These ensure that the network will continue to be available and offer the users quality of service without serious interruption or failure. It was designed to expand to include new user groups and remote sites. It can also support new applications without impacting the level of service delivered to existing users. Point to multi-point wireless infrastructure was adopted in the access layer. The network offers point-to-multipoint connection enabling more network users to be connected to Internet services. It allows for expansion of the network as a result of ptMP design, because additional network equipment can easily be added into the network and expand its scope of operation to cover more users. Other sustainability measures include availability, security and manageability.

The wireless network was designed and implemented to deliver consistent, and reliable performance and available for the users to use. In addition, the failure of a single link or piece of equipment will not significantly impact network performance. Alternatively solar power supply was provided as the primary source of power to power the equipment, while electricity from the national grid is used as the secondary source of power. At the access layer, the network has been scaled up into a mesh network of access point to reduce attenuation from environmental obstacles. The OSPF routing protocol was configured to enable easy recalculation of a backup route in the event of a downtime at any route. During the period of observation and monitoring, the network down time was reduced seriously and the performance of the equipment and availability of the network was optimal. The cumulative downtime within the first 3 years did not exceed 40 hours.

A network that is too complex or difficult to maintain cannot function effectively and efficiently. The operating centre of the Network was setup to monitor and manage the devices and the entire network system. During the first 12 months, it was very easy to monitor and manage the entire network and devices. Operational issues were promptly monitored and reported using the network monitoring software known as Dude. Very few problems were observed and reported. Issues like bandwidth bottleneck was observed and promptly resolved through bandwidth shaping. The security of the wireless network has been scaled upward with the addition of the IEEE 802.11i standard to secure data transmission between the user end devices and access points.

Security is a feature that must be designed into a network. Planning the location of security devices, filters, and firewall features is critical to safeguarding network resources. MD5 level of password was adopted for all equipment. This device is a proprietary device for performing authentication, authorization and access control functions on the wireless network. Every wireless device in the network was given a password using an MD5 level of encryption. The radius server was setup at the core layer to prevent unauthorized access and to allow only authorized users have access to the wireless network.

\section{Conclusion}

Wireless networks are very good and affordable means of providing Internet connectivity for rural communities. The environment can sometimes pose as a challenge as a result of natural obstacles that can emanate after deployment. But with a scalable network design this challenge can be surmounted. The introduction of the mesh network on the access layer using the controlled access point system manager (CAPSMAN) reduced signal attenuation as a result of new obstacles along the path of transmission. In the study, we made effort to optimize the wireless network using the cisco hierarchical network model and added point-to-multipoint network to the access layer. In order to sustain network availability to the users, we set all devices on the network to obtain alternate source of power using solar power should in case the national power grid fails. During deployment, it was initially difficult to get the cooperation of the community members to permit the mounting of the equipment in their vicinity without financial reward. But with adequate explanation and dialogue they understood the benefits and accepted without financial reward.

In order to implement affordable, reliable and secure wireless network, we explored different technologies such as the open shortest path first (OSPF) layer 3 algorithm and created a virtual private network (VPN) using the layer 2 technology, Ethernet Over Internet Protocol (EOIP) on affordable wireless network devices. We have implement a wireless network architecture with an access layer organized into a mesh network of access points which has greatly reduced signal interference from obstacles such as trees in the environment. The access network is placed closer to the client premises equipment (CPE) to reduce the distance between access points and end user devices and improve signal quality. We were able to create a point-to-multipoint connection in five locations in the rural communities using the Off-the-shelf 802.11n MIMO chipset. This has tremendously reduced signal transmission interference from obstacles. A 1.5 Mbps used in the network showed 
a $0.5 \mathrm{Mbps}$ signal loss indicating a high throughput on the network with quality of service to the end users. In the network, we have observed good number of users/devices accessing the network and a higher number is possible.

\section{References}

[1] Atel Electronics (2015): Wireless access point Airmax TDMA (Ubiquiti NanoStation M5) Accessed from: http://www.atelelectronics.eu/produkt.php?hash=07006 (Accessed: March 15, 2017).

[2] Brewer, E., Demmer, M., Ho, M., Honicky, R. J., Pal, J., Plauch, E. M., and Surana, S. (2006). The challenges of Technology research for Developing Regions, Institute of Electrical and Electronic Engineers Pervasive Computing, 5(2), 15.

[3] Disraeli, B, (2001) Information Technology for the Development of Youth in Africa. Retrieved from http//www.ayf.de/documents/dixit.doc.

[4] Edim, A. E., Muyingi, H. and Sibanda, K. (2013). A voice user Interface for Low-literacy Users in a Rural Community. Journal of Computing and ICT Research. 7(1).

[5] Divya, S. (2011). A Low-Cost Efficient Wireless Architecture for Rural Network Connectivity. Electronic Project. Copyright Classle. Retrieved from: https://www.cs.nyu.edu/ lakshmi/wire.pdf

[6] Du Bowei, Demmer, M. and Brewer, E. (2006). Analysis of WWW Traffic in Cambodia and Ghana. 15 thinternational WWW Conference (May 2006). Edinburgh.

[7] Dymond A., and Oestman, S. (2008) Output-Based Aid in Mongolia: Expanding Telecommunication Services to Rural areas. World Banbk Washington DC.

[8] Flickenger, Rob., Aichele Corinna., Fonda Carlo., Forster Jim., Howard Ian., Krag Tomas., and Zennaro Marco. (2006). Wireless Networking in the Developing World. Limehouse Book Sprint Team, First Edition.

[9] Galperin, Herman. and Bar, Francois. (2004). Building the Wireless Internet Infrastructure: from cordless Ethernet Archipelagos to Wireless grids. Communication and Strategies, 54(2), $45-68$.

[10] Gast, M.S. (2002). 802.11 Wireless Networks: The Definitive Guide (1st ed.). Sebastopol: O’Reilly \& Associates, Inc.

[11] Horst, H. and Miller, D. (2006). The Cell Phone: An Anthropology of Communication. Berg, 2006.

[12] IEEE (2007) Wireless LAN Medium Access Control (MAC) and Physical Layer (PHY) Specifications. IEEE Standard for Information Technology- Telecommunications and information exchange between systems. Accessed from: http://www.ie.itcr.ac.cr/acotoc/Ingenieria/Lab\%20TEM\%20II/Antenas/Especificacion\%20802\%2011-2007.pdf (Accessed April 20, 2016).

[13] Imran A. U., and Liaquat A. T. (2007) Implementing Wireless Infrastructure Network with Efficient Security, In: Proceedings of the $9^{\text {th }}$ Islamic countries conference on statistical sciences.

[14] Kumar, A., Rajput, N., Chakraborty, D., Argarwal, K. S., and Nanavati, A. A. (2007). WWWTW: The World-Wide Telecom Web. In: NSDR'09 Kyoto, Japan.

[15] LitePoint (2012): LitePoint Introduces IQxel-The First 802.11ac Manufacturing Test Solution. Accessed from: http://litepoint.com/press-releases/litepoint-introduces-iqxel-the-first-802-11ac-manufacturing-test-solution/ (Accessed: March 12, 2016).

[16] Long Z., Song X., Zhang L., Xiao Y. (2012) Design and Implementation of Wireless Local Area Network Videophone. In: Sambath S., Zhu E. (eds) Frontiers in Computer Education. Advances in Intelligent and Soft Computing, vol 133. Springer, Berlin, Heidelberg. https://doi.org/10.1007/978-3-642-27552-4 62

[17] Mishra, S. M., Hwang, J., Filipini, D., Du, T., Moazzami, R., and Subramanian, L. (2005) Economic Analysis of Networking Technologies for rural Developing Regions, In: Proceedings of $1^{\text {st }}$ Workshop on Internet and Network Economics (WINE), December 2005.

[18] Nadevschi, S., Surana, S., Du, B., Patra, R., Brewer, E., and Stan, V. (2007). Potential of CDMA450 for Rural Network Connectivity. Institute of Electrical and Electronic Engineers Communication Magazine. Special Issue on new Directions in Networking Technologies in Emerging Economics.

[19] Netgear (2005). Netgear Annual Report 2005. Accessed 2017. Accessed from: http://annualreports.com.

[20] Nikos, K. and Kyriakos, V. (2010). Building Wireless Metropolitan Networks. Research Academic Computer Technology Institute and Computer Engineering and Informatics Department, University of Patras.

[21] Noll, G. Roger (2000). Telecommunications reform in developing countries. In A. O. Kruger (Ed). Economic policy reform: The second stage. Chicago, University of Chicago Press.

[22] Patel, Neil., Chittamuru, Deepti., Jain, Anupam., Dave, Paresh. and Parikh S. Tapan (2010). Avaaj Otalo - A Field Study of an Interactive Voice Forum for Small Farmers in Rural India. In: CHI'10, Atlanta, GA.

[23] Patra K. R., Nedevschi, S., Surana, S., Sheth, A., Lakshminarayanan, S., and Brewer, E. A. (2007): WiLDNet: Design and Implementation of High Performance WiFi Based Long Distance Networks. NSDI 1(1).

[24] Pentland, A. Fletcher, R., and Hassan, A. A. (2004). DakNet: Rethinking Connectivity in Developing Nations. Computer 37(1): 78-83.

[25] Sonesh, S., Rabin K. P., Sergiu, N., Manuel, R., Lakshminarayanan, S., Yahel B., Eric A. B. (2008). Beyond Pilots: Keeping Rural Wireless Networks Alive. NSDI 8,119-132.

[26] Subramanian, L., Surana, S., Patra, R., Ho, M., Sheth, A., and Brewer, E. (2006). Rethinking Wireless for the Developing Wprld. HotNets- $V$

[27] Todd L. (2007). Cisco Certified Network Associate. $6^{\text {th }}$ Ed, Wiley Publishing Inc., Indianapolis, Indiana.

[28] Toni A. and Wendy G. P. M., (2007) Design and Implementation of a Wireless Network System in a Smart Campus, Communication and Information Technology Journal 1(2). 127-139

[29] Wallsten, J. S. (2003). Regulation and Internet use in Developing Countries. Washington DC, AEI-Brookings WiFi's Growing Pains (Special Report). 2004, February 18). Business Week Online. Retrieved May 18, 2018. (http://www.businessweek.com/technology/tcspeccial/tc-04wi.htm)

[30] World Bank Report (2004). World Development indicators. Washington, DC: World Bank.

[31] Yunus, M. (1999). The Grameen Bank. Scientific American 


\section{Authors Profile}
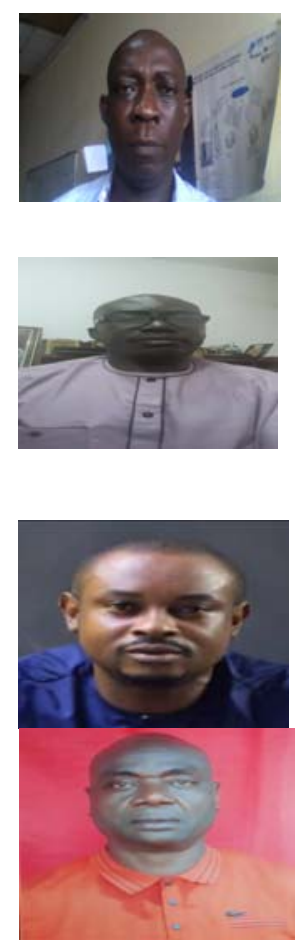

Edim Azom Emmanuel is a Computer Scientist and a Associate Professor in the Department of Computer Science, University of Calabar. He Holds an M.Sc. and Ph.D in Computer Science. His interest include ICT, computer networking and software engineering

Ogban Felix Ukpai is a Computer Scientist and a senior lecturer in the Department of Computer Science, University of Calabar. He Holds an M.Sc. and Ph.D in Computer Science. Interest include computer networking and computer algorithms

Okoro Osahon is an Electronic Engineer in the Department of Computer Science, University of Calabar. $\mathrm{He}$ is a Computer Networking expert with professional qualifications in CCNA. He holds a Master degree in Computer Science.

Ofem Ajah Ofem is a Computer Scientist and a senior lecturer in the Department of Computer Science, University of Calabar. He Holds an M.Sc. and Ph.D in Computer Science. Interest include Software Engineering and algorithms 